\title{
ASPECTOS RELEVANTES NA AVALIAÇÃO DE ELETRODOS PARA FORNOS ELÉTRICOS A ARCO*
}

\author{
Paulo von Krüger ${ }^{1}$ \\ Gustavo von Krüger ${ }^{2}$ \\ Niveo Roberto Campos e Souza ${ }^{3}$
}

\begin{abstract}
Resumo
Nas condições adversas do mercado atual, aliadas à exacerbada competição é uma questão de sobrevivência a otimização de resultados. Para tal é imprescindível que os principais insumos, particularmente os mais gravosos sejam criteriosamente selecionados. Os eletrodos de grafite estão entre os itens principais na operação dos fornos elétricos a arco, tanto do ponto de vista técnico, quanto econômico e a escolha correta deste insumo deve contemplar aqueles dois enfoques. Esta escolha se faz dentre as diversas classes, disponibilizadas no mercado: RP, HP, SHP, UHP. $O$ que se pretende no presente trabalho é sugerir uma metodologia que, contemplando o elenco de parâmetros influentes, permita fazer a escolha correta do eletrodo, dentro daquelas classes, tanto sob o enfoque técnico, quanto econômico.

Palavras-chave: Eletrodos; Fornos elétricos a arco.
\end{abstract}

\section{RELEVANT FEATURES ON THE EVALUATION OF ELECTRIC ARC FURNACE ELECTRODES}

\section{Abstract}

In the tight and competitive market conditions, the optimizing of results is a matter of survival. On this purpose, it is mandatory that the main inputs, chiefly the more costly ones, must be cautiously selected. Graphite electrodes are among the main inputs on electric arc furnaces operations, both on the technical and the economical poin of view and its choice must consider those two approaches. This choice is carried out among the several electrodes classes available in the market: RP, HP, SHP, UHP. This work intends to propose a method that considers the whole of influent parameters and leads to the suitable choice, among those classes, both on technical and economical point of view.

Keywords: Electrodes; Electric arc furnaces.

1 Engenheiro de Minas e Metalurgia, MSc Diretor Técnico da ACK Representantes Ltda Professor. aposentado da Escola de Minas/UFOP, Membro da ABM, TMS, AISTECH, ISIJ, MG, Brasil. Administrador Diretor Executivo da ACK Representantes Ltda., MG, Brasil.

Engenheiro Metalurgista, Assistente Técnico ACK Representantes Ltda., MG, Brasil. 


\section{INTRODUÇÃO}

Os eletrodos de grafite estão entre os itens principais na operação dos fornos elétricos a arco, tanto do ponto de vista técnico, quanto econômico.

Assim sendo, é de todo desejável que os critérios adotados na sua seleção, sejam os mais precisos possíveis.

Com efeito, um eletrodo sub especificado leva a um aumento de consumo e comprometimento na produtividade, decorrente de uma frequência maior de paradas para acréscimos às colunas.

Por outro lado, uma super especificação leva ao uso desnecessário de um material mais caro, onerando o produto.

Como se pode intuir, ambos os tipos de restrições são relativamente voláteis, já que dependem de fatores antagônicos - a produtividade tende a crescer com a qualidade e vice versa; a qualidade e o preço do eletrodo crescem e decrescem no mesmo sentido.

Uma escolha correta implica, portanto em uma compatibilização daqueles fatores, visando a otimização do resultado final, refletido no custo do produto.

O que se pretende no presente trabalho é sugerir uma metodologia que, contemplando o elenco de parâmetros influentes, permita fazer a escolha correta do eletrodo, tanto sob o enfoque técnico, quanto econômico

\section{CONSIDERAÇÕES INICIAIS}

Como se sabe, os eletrodos são divididos em classes, de acordo com as suas propriedades, visando conferir flexibilidade na sua seleção, em função das condições operacionais às quais eles serão submetidos. Com isto, criam-se condições de compatibilidade, tanto técnica, quanto econômica, entre o insumo e a operação.

A cada classe de eletrodo corresponde um elenco de procedimentos de fabricação e formulações de misturas de componentes. Estes incluem a seleção, preparação e formulação dos componentes, as condições de cozimento, impregnação, grafitização e usinagem.

Dente estes, a qualidade das matérias primas, especificamente dos coques têm uma influência decisiva nas propriedades do produto. De fato, o tipo de coque, juntamente com as características geométricas das partículas, determinam a capacidade de condução de corrente e as propriedades mecânicas do eletrodo.

Os eletrodos de grafite são fabricados empregando uma gama relativamente alta de tipos de coque de petróleo, que vão desde o coque anódico (assim denominado por ser utilizado na fabricação de anodos para a indústria de alumínio), até o coque acicular (needle coke) de última geração.

É escusado dizer que quanto mais alto o grau do coque empregado, melhores as propriedades elétricas (resistividade), mecânicas (resistência à flexão,...) e térmicas, Em função das proporções dos diversos tipos de coque empregados em cada formulação, são produzidos diferentes tipos de eletrodo (classes).

Estas classes podem ter denominações diversas, em função do fabricante, mas para efeito deste trabalho será adotada a nomenclatura que se segue, reconhecida mundialmente.

Segundo ela, os eletrodos se classificam em Regular Power (RP), High Power (HP), Super High Power (SHP) e Ultra High Power (UHP).

Simplificadamente, as características de cada classe, são mostradas na Tabela 1. 
Tabela 1 - Propriedades típicas dos eletrodos, por classe

\begin{tabular}{|c|c|c|c|c|}
\hline \multirow{2}{*}{ Propriedades } & \multicolumn{4}{|c|}{ Classe do eletrodo } \\
\hline & $\mathrm{RP}$ & $\mathrm{HP}$ & SHP & UHP \\
\hline $\begin{array}{l}\text { Resistividade elétrica } \\
\mu \Omega m\end{array}$ & $\begin{array}{l}7,0 \quad- \\
11,0\end{array}$ & $6,5-7,5$ & $4,8-6,0$ & $4,0-5,5$ \\
\hline $\begin{array}{l}\text { Resistência a flexão - } \\
\mathrm{MPa}\end{array}$ & $6,5-15$ & $10-18$ & $10-18$ & $10-18$ \\
\hline $\begin{array}{l}\text { Densidade aparente - } \\
\mathrm{g} / \mathrm{cm}^{3}\end{array}$ & $\begin{array}{l}1,55 \\
1,67\end{array}$ & $\begin{array}{l}1,65 \\
1,76\end{array}$ & $\begin{array}{l}1,65 \\
1,78\end{array}$ & $\begin{array}{l}1,65 \\
1,78\end{array}$ \\
\hline $\begin{array}{l}\text { Coeficiente de expansão } \\
\text { térmica }\left(20-520^{\circ} \mathrm{C}\right), 1^{-} \\
6 / \mathrm{K}\end{array}$ & 2,8 & 2,2 & 1,7 & 1,5 \\
\hline
\end{tabular}

Há que se ressaltar que os valores mostrados, são indicativos, usados como referência nesta apresentação. Os valores reais podem divergir, em função das alternativas do fabricante, do diâmetro do eletrodo e, ainda, de condições operacionais específicas. Naturalmente, isto não invalida a metodologia apresentada adiante.

Como se pode observar, dentre os parâmetros selecionados, à exceção da classe $\mathrm{RP}$, os dois que têm diferenças relevantes são a resistividade elétrica e o coeficiente de expansão térmica.

No que se refere a este último, o que importa é a seleção correta do nipple. Em princípio, poder-se-ia sugerir que maiores coeficientes de expansão térmica aumentam a probabilidade de juntas abertas e despontes, mas não há como afirmar se isto ocorrerá ou não.

Já o valor da resistividade tem influência direta no consumo de energia e no do eletrodo em si. Aliás, esta é a razão de ser das diferentes especificações dos eletrodos. Em função dos programas de potência adotados na operação, são estabelecidos, ainda no projeto, os diâmetros e recomendadas as resistividades desejáveis, ou seja, a classe do eletrodo.

O valor ideal da resistividade é relativamente volátil e varia dentro de faixas, características de cada classe. Estas faixas são, em última análise, fruto de uma análise custo-benefício baseada em dados operacionais, quando são estabelecidos limites, superior e inferior, para cada diâmetro de eletrodo. Na realidade, trata-se de um balizamento para sugerir os campos de aplicação de cada classe.

A figura 1 mostra as zonas recomendadas para o emprego de cada classe, em função do diâmetro (dado de projeto) e corrente (dado de operação). Na realidade, elas encerram, em uma primeira aproximação, as recomendações do fabricante, quanto ao uso de seus produtos.

A título de ilustração, é mostrado o exemplo de um eletrodo de $450 \mathrm{~mm}$ de diâmetro, submetido a uma corrente de $36 \mathrm{kA}$. Como mostrado, o eletrodo recomendado seria um SHP.

Apesar de tecnicamente correta, esta indicação é, até certo ponto, engessada, já que não leva em consideração todas as variáveis envolvidas.

$\mathrm{Na}$ desenvolvimento, a seguir, tomando como referência a indicação do gráfico da Figura 1, serão avaliadas e quantificadas as implicações da adoção de soluções diferentes daquela. 


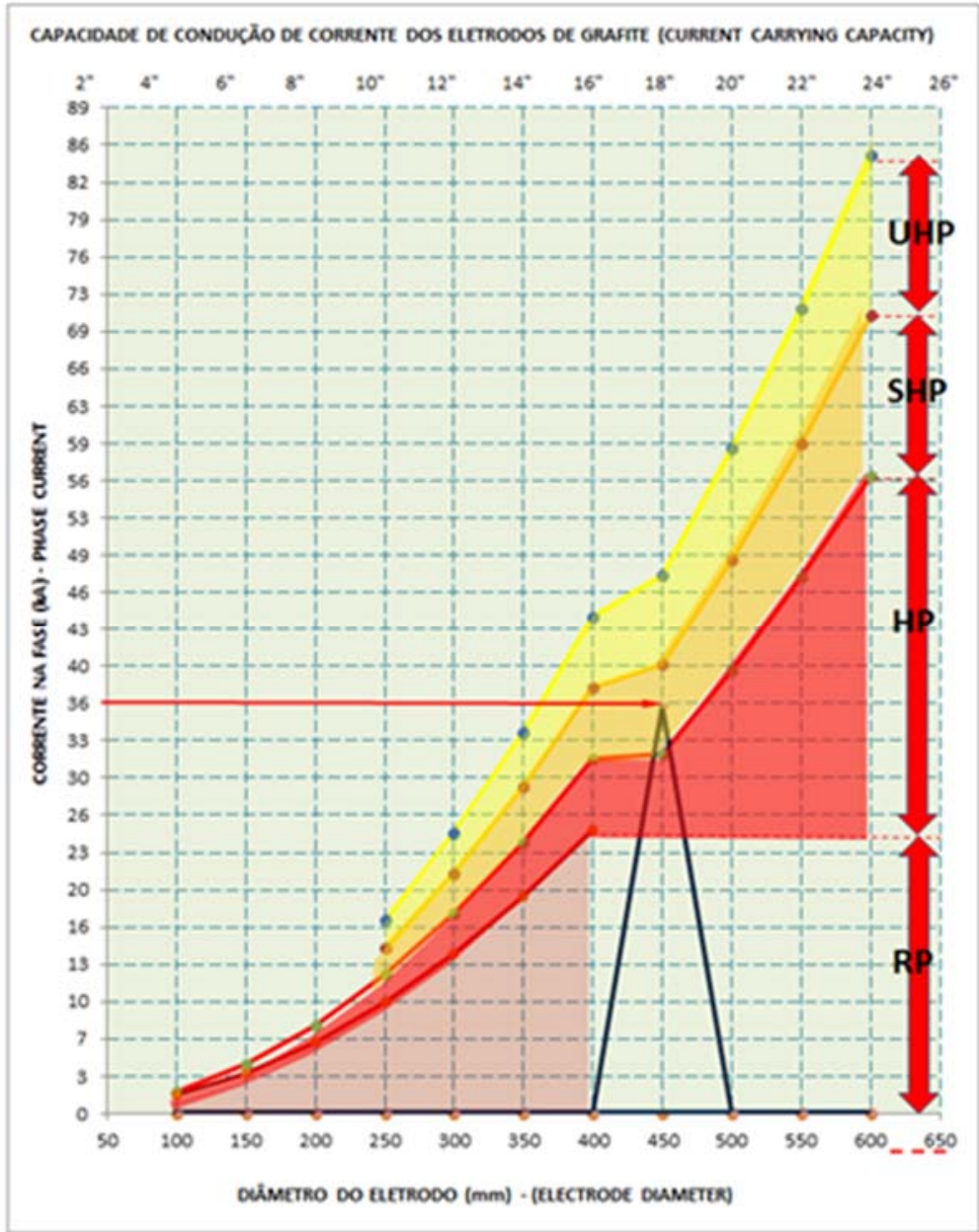

Figura 1 - Classe do eletrodo em função da corrente e diâmetro

\section{FATORES INFLUENTES - CARACTERIZAÇÃO E AVALIAÇÃO}

A título de ilustração, as condições de referência mostradas na figura 1, isto é, um eletrodo com $450 \mathrm{~mm}$ de diâmetro e uma corrente (média) de $36 \mathrm{kA}$, sinalizam para um eletrodo da classe SHP.

Se for feita a opção para uma classe acima (UHP), a coluna admitiria correntes acima de $40 \mathrm{kA}$, o que implica que para a corrente de $36 \mathrm{kA}$, o valor da potência de perda será menor, o que leva a um menor consumo de energia e maior produtividade. Adicionalmente, a coluna vermelha é menor, em relação à com SHP, o que resulta em um menor consumo de eletrodos e uma menor frequência de montagens.

Já na hipótese de se optar por uma classe abaixo (HP), onde a corrente máxima recomendada seria de $32 \mathrm{kA}$, a aplicação da corrente de $36 \mathrm{kA}$ leva a um maior valor da potência de perda, com o consequente aumento do consumo de energia e perda de produtividade. Neste caso, a coluna vermelha será maior, resultando em aumento de consumo e aumento da frequência das montagens.

Partindo do princípio que uma alternativa, qualquer que seja, será válida se esta implicar em uma redução do custo de produto final, no caso, do aço. Assim sendo, na primeira hipótese, o maior preço do eletrodo, em relação ao de referência (SHP, no exemplo), deverá ser compensado pelo valor dos ganhos relacionados. Do mesmo modo, na segunda opção, o menor preço do eletrodo deverá ser suficiente para compensar as perdas. 
No seguimento são analisados os itens acima relacionados.

\subsection{Potência de perda}

Esta corresponde à perda decorrente da resistência do condutor. Ela pode ser desmembrada nas perdas do circuito secundário até as placas de contato e nas correspondentes ao condutor eletrodo. A primeira parcela é constante, independente da classe do eletrodo, enquanto a segunda varia com a resistividade da coluna.

$\mathrm{Pp}=P p_{\mathrm{c}}+\mathrm{Pp}$,

O valor da perda na coluna será:

$\mathrm{Pp}_{\mathrm{e},}=\rho \cdot \mathrm{L} /\left(\pi \mathrm{D}^{2} / 4\right)$,

onde,

$r$ - resistividade do eletrodo; $L$ - comprimento da coluna abaixo da garra; diâmetro médio do eletrodo

Chamando de $\rho 0$ a resistividade do eletrodo da classe de referência e $\rho_{1}$ a da opção a ser avaliada, a diferença entre as potências de perda, será:

$\Delta P p=\left(\rho_{0}-\rho_{1}\right) \cdot L /\left(\pi D^{2} / 4\right) \mathrm{kWh} / \mathrm{h}$

Que será negativa se a nova classe for inferior à de referência e positiva se ela for superior.

Esta diferença implica em uma variação, positiva ou negativa do consumo de energia elétrica e, respectivamente no aumento ou redução do ciclo do forno (tap to tap).

Sendo,

$\mathrm{Pu}=\mathrm{Pw}-\mathrm{Pp}$,

A produtividade do forno será, na referência,

$\mathrm{B}_{0}=(\mathrm{Pw}-\mathrm{Pp}) / \mathrm{Q} \mathrm{t} / \mathrm{h}$

e na alternativa,

$\mathrm{B}_{1}=(\mathrm{Pw}-[\mathrm{Pp}+\Delta \mathrm{Pp}) / \mathrm{Q} \mathrm{t} / \mathrm{h}, \mathrm{e}$

$\Delta B=\left(B_{0}-B_{1}\right) t / h$

$Q$ é o consumo específico de energia elétrica $\mathrm{kWh} / \mathrm{t}$.

O valor monetário destas variações será

$\$ M=\Delta P p . \$ W+\Delta B . \$ A \cdot a$

onde

\$M é o valor monetário das variações, \$W é o custo da energia elétrica, \$A é o valor comercial do aço e "a", a margem de lucro da comercialização.

\subsection{Consumo de eletrodo}

Além dos fatores acima, há que se considerar que a variação da resistividade leva a uma variação do consumo do eletrodo pela oxidação lateral.

Com efeito, como se sabe, o consumo contínuo de eletrodos em um forno elétrico a arco é dividido em dois componentes, a saber, o consumo da ponta e o consumo lateral.

Enquanto o consumo e ponta é função, essencialmente, do valor da corrente, o da lateral depende, dentre outras variáveis, da área da coluna com temperatura acima da temperatura de reação do carbono do eletrodo (coluna vermelha).

Há várias expressões que procuram calcular estes consumos. As duas, mostradas a seguir, mostram aquela dependência. As parcelas correspondentes ao consumo da ponta, foram omitidas deliberadamente, a bem da simplicidade.

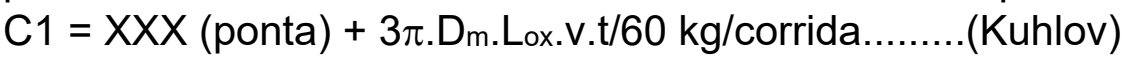


$\mathrm{C} 2=\mathrm{XXX}($ ponta $)+\left[\mathrm{NE}_{\mathrm{E}}\right.$ Ls. $\left.\pi . \mathrm{D}_{\mathrm{m}}\right] \cdot \mathrm{Fs} .(\mathrm{t}) / \mathrm{G}_{\mathrm{A}} \mathrm{kg} / \mathrm{taço} \ldots . . .($ Bowman/CE)

Dentre as variáveis, pode-se destacar o comprimento da coluna vermelha e o tempo total de ciclo (tap to tap). A primeira encerra a resistividade elétrica, como se verá em seguida.

A Figura 2 mostra os fluxos de calor no entorno do eletrodo.

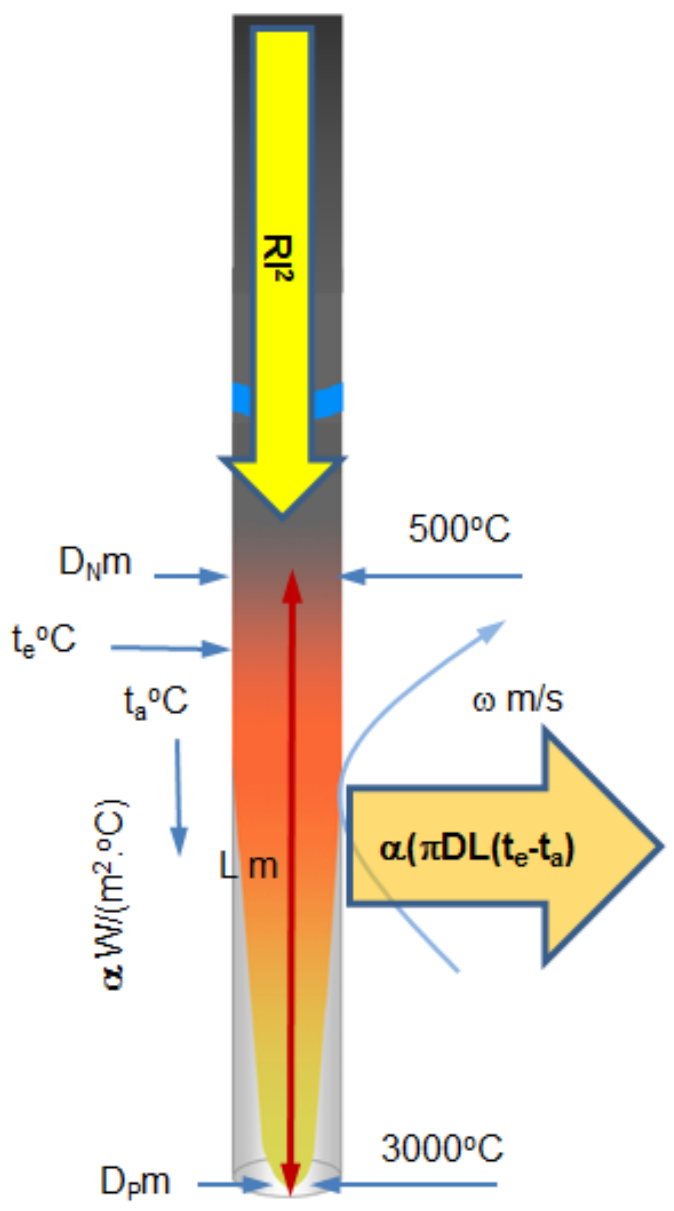

Figura 2 - Fluxo de calor numa coluna de eletrodos em FEA

A energia gerada pela passagem da corrente, ou seja,

$\mathrm{RI}^{2}=\rho \mathrm{L} /\left(\pi \mathrm{D}^{2} / 4\right)$

(equação 2)

deve estar em equilíbrio com a dissipada na superfície da coluna:

$\mathrm{J}=\alpha \mathrm{S} \Delta \mathrm{t}=\alpha \cdot \pi \mathrm{DL}\left(\mathrm{t}_{\mathrm{e}}-\mathrm{t}_{\mathrm{a}}\right)$

onde,

a - coeficiente de transmissão de calor do meio circundante

$S$ - superfície de dissipação; D - diâmetro; $L$ - comprimento

Naturalmente, se o fluxo de saída de calor ficar menor do que o aporte, para se restabelecer o equilíbrio, a temperatura do corpo gerado se eleva e/ou a superfície de dissipação aumenta.

A rigor, a superfície de dissipação é toda a coluna, sendo, portanto, invariável. Assim sendo, o que ocorre é um aumento de temperatura, de modo a se aumentar o fluxo de saída. Naturalmente, na condição inversa, isto é, a redução do aporte de calor, a temperatura da coluna diminui. Como se observa na figura 2 , a temperatura é variável ao longo da coluna e, assim, o aumento da temperatura se dá pelo deslocamento das isotermas no sentido ascendente e, no caso de diminuição, no 
sentido descendente. Ainda na Figura 2, está assinalada a isoterma de $500^{\circ} \mathrm{C}$. Esta temperatura é considerada como o limite, a partir do qual o carbono do eletrodo reage com o oxigênio, provocando o consumo lateral. O segmento da coluna a partir desta isoterma é a denominada "coluna vermelha". Assim, resumindo, um maior aporte de calor leva à subida da isoterma de $500^{\circ} \mathrm{C}$ e uma redução daquele aporte, a um abaixamento da mesma isoterma, ou, em outras palavras, respectivamente, ao aumento ou diminuição da coluna vermelha.

Isto é mostrado, ilustrativamente, na Figura 3.
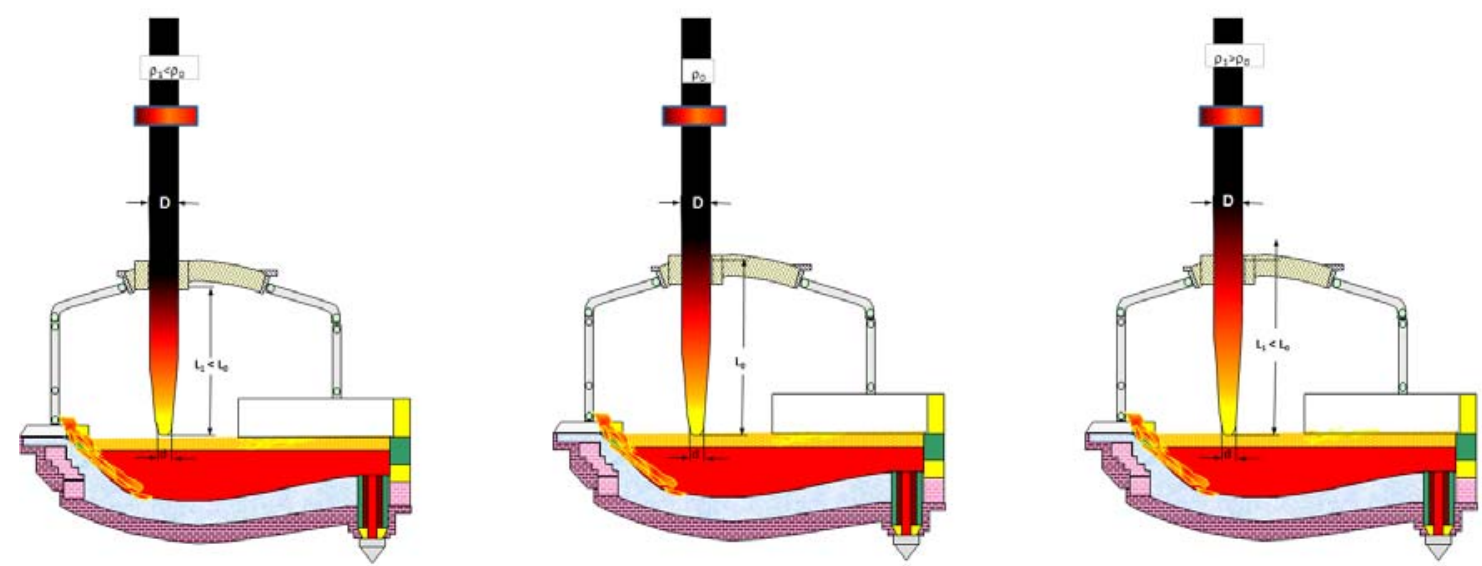

Figura 3 - Variação da coluna vermelha com a resistividade do eletrodo

A partir do balanço de fluxos, pode-se calcular a variação do tamanho da coluna vermelha, em relação à referência.

Levando os valores determinados nas expressões que representam o consumo lateral, ter-se-á a variação do consumo, positiva ou negativa, conforme o caso.

Exemplificando, considerando o valor $L_{1}$ (ver figura 3 ), calculado para o eletrodo alternativo e sendo Lo, o de referência, e usando a fórmula de Kuhlov (equação 9), a variação de consumo lateral será:

$3 \pi . D_{m} .\left(L_{0}-L_{1}\right) \cdot v . t / 60 \mathrm{~kg} /$ corrida

Sendo \$E o preço do eletrodo de referência, o valor desta variação será

$3 \pi . D_{m} .\left(L_{0}-L_{1}\right) \cdot v \cdot t / 60 . \$ E / c o r r i d a$

Uma última variação a ser considerada seria a da frequência de montagens das peças, decorrente da variação do consumo.

A relevância desta variação depende das condições de operação.

Duas hipóteses são consideradas.

Na primeira, a montagem das peças é feita no horário de pico, quando o forno está desligado. Neste caso não há perda de temperatura, decorrente da troca, nem perda de produtividade. Eventuais montagens no horário de operação seriam de caráter eventual, não causando um efeito a ser levado em consideração.

No caso de uma operação direta, sem parada sazonal, o maior número de trocas deveria ser contemplado. Sugere-se que se adote um fator de correção, com base no histórico da operação. Um cálculo mais preciso é precário, já que são ações pontuais. A título de ilustração, o consumo adicional de energia em uma troca seria, conservadoramente, similar a um carregamento de cesto. Neste caso, o consumo adicional de energia estaria em torno de $10 \mathrm{kWh} / \mathrm{t}$. 


\section{VALOR DA SUBSTITUIÇÃO}

O valor total da substituição do eletrodo de uma classe para o de outra (superior ou inferior), seria a soma dos valores referentes aos diversos fatores, relacionados anteriormente.

Sendo GA, o peso de aço por corrida, o valor monetário das variações é a soma da equação (8) com a 13, referidas a um dia de operação:

$\$ V=\left[(\Delta P p . \$ W+\Delta B . \$ A . a) \cdot h+3 \pi \cdot D_{m} \cdot\left(L_{0}-L_{1}\right) \cdot v \cdot t / 60 / G_{A} . \$ E . N\right] \$ / d i a$

Sendo $\mathrm{h}$, o número de horas de produção por dia e $\mathrm{N}$ o número de corridas no mesmo período.

A referência à unidade dia se deve ao fato que a produtividade ser melhor expressa em unidades de tempo. A rigor, quanto maior o período considerado, mais precisos serão os resultados.

É claro que esta expressão pode ser referida à tonelada de aço, ou qualquer outra base, bastando, para tal fazer a devida conversão.

Conhecendo-se os consumos diários de eletrodos, estimados, por exemplo, pela equação (9) e aplicando-se a este valor o preço do eletrodo de referência, tem-se a despesa diária com o eletrodo atual

$\$ C_{\text {ref }}=\left[X X X(\right.$ ponta $)+3 \pi . D_{m} . L_{o} . v . t / 60 \mathrm{~kg} /$ corrida). $\mathrm{N}$ corridas. \$E] \$/dia

A despesa diária com o eletrodo alternativo seria, então,

$\$ \mathrm{C}_{\text {alt }}=\$ \mathrm{C}_{\mathrm{ref}}+\$ \mathrm{~V}$

Assim sendo, o preço limite do eletrodo alternativo, para ter os mesmos resultados do de referência deverá ser

$\$ \mathrm{C}_{\text {alt }}=\left[\$ \mathrm{C}_{\text {ref }} /\left(\$ \mathrm{C}_{\mathrm{ref}}+\$ \mathrm{~V}\right)\right] .100 \%$ de $\$ \mathrm{C}_{\text {ref }}$

\section{CONSIDERAÇÕES FINAIS}

Sendo os eletrodos um dos principais itens do custo de produção de aço em fornos elétricos a arco, a sua correta seleção, compatibilizando fatores conflitantes, como índices de desempenho e valor comercial (elevado) deste insumo, requer que na avaliação das alternativas de fornecimento, sejam consideradas todas as variáveis relevantes.

O presente trabalho pretendeu apresentar um critério de avaliação preciso e funcional, que permite identificar o valor real de uma alternativa, em relação à referência em uso do modo a avaliar o mérito da oportunidade.

As variáveis envolvidas são as disponíveis na usina ou facilmente calculáveis.

\section{REFERÊNCIAS}

1 Energoprom Group. Informativo da Empresa. http://www.energoprom.ru/en/

2 Borlée J, Wauters M, Weber M. Monitoring system for controlling and reducing the electrode consumption in DC EAF plants. European Comission. Disponível em bookshop.europa.eu/.../monitoring-system-for-contr

3 Cardoso, José J. Eletrotermia Fornos Elétricos a Arco. Imprensa da Universidade Federal de Ouro Preto; 1987

4 Kuhlow, P. Graphite Electrodes - Potential for Reduction and Consumption. Sonderdruck aus „elektrowärme international. 61. Jahrgang, Heft 3, September 2003 • Pgs 117-124 • Vulkan-Verlag • Essen

5 ACK Representantes - Informações internas 